matées par l'amas de plantes décomposées. Rien ne s'accrochait aux pales fuyantes de l'hélice (Fig. 29).

Seule la marche arrière est impossible dans les herbes.

Le garde particulier auquel appartient ce bateau, habite Saint-Philbertde-Granlieu. Il était obligé autrefois, lorsqu'il ne disposait que d'une hélice ordinaire, de renoncer à naviguer pendant tout l'été, parce qu'il lui faut,

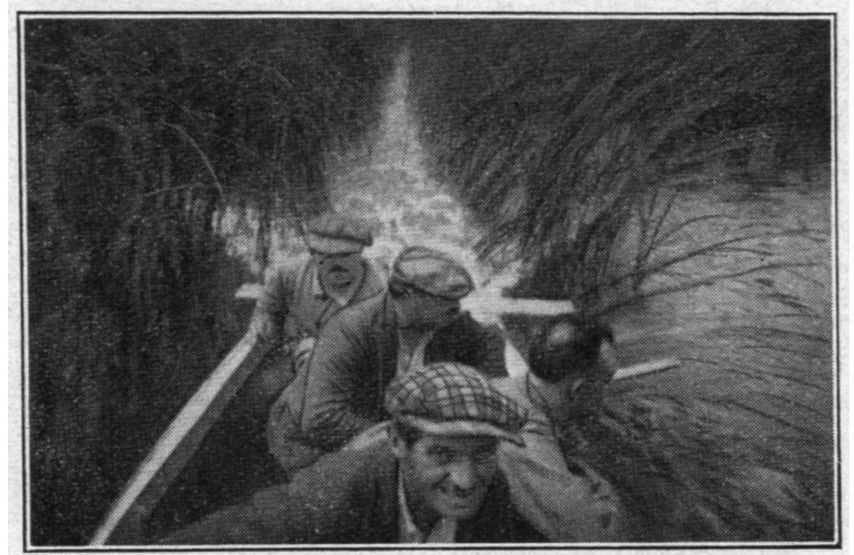

Fic. 29. - Frayée ouverte dans les joncs du lac de Granlieu, par une barque propulsée par l'hélice anti-herbes.

pour gagner la partie libre du lac, descendre la rivière La Boulogne, ellemême complètement envahie par la végétation, puis traverser la partie du lac transformée en marais. Maintenant, il peut circuler partout, en tout temps, et avec le sourire, ainsi qu'en témoigne la photo ci-jointe.

Cette nouvelle hélice pourrait rendre service aux propriétaires d'étangs et être utilisée à la propulsion des bateaux faucardeurs. Ajoutons, pour terminer, qu'elle semble avoir un excellent rendement en eau libre, car le mème bateau gagne plusieurs kilomètres à l'heure depuis qu'il en est muni ; toutefois, aucune expérience au dynamomètre n'a encore été faite à ce sujet.

\title{
A PROPOS DES PESTES D'ÉCREVISSES
}

\section{Par M. DE DROUIN dE BOUVILLE}

Les mortalités qui, depuis une soixantaine d'années, ont anéanti ou raréfié à l'extrême les Ecrevisses dans presque toutes les eaux douces d'Europe, ont fait couler des flots d'encre. Et la source n'en est pas tarie comme on a pu en juger par la toute récente étude du Professeur Schaprrclaus (I).

(1) La cause des pestes d'Ecrevisses ; - Bulletin, no $n^{\circ}$ 1o, Octobre 1936, p. 85 ; $n^{\circ}$ ror, Novembre 1936, p. 117 . 
Mais que penser au juste, après lecture de ce travail, qui rouvre la discussion, des théories antérieures qui ont incriminé successivement, comme cause d'une maladie épidémique dite peste, des parasites. fort variés.

En 19o6, après plusieurs années consacrées à des recherches tendant au repeuplement des ruisseaux où la pêche aux balances était jadis si fructueuse, nous écrivions que l'agent pathogène ne pouvait être qu'un Bacille, un Sporozoaire ou un Champignon ( $\mathrm{I}$ ).

Après discussion, la conclusion était que, conformément aux vues du Professeur Horkr, de Munich, alors dans tout l'éclat de sa réputation, il convenait de voir dans la peste une infection bactérienne.

Notre préférence raisonnée s'est trouvée singulièrement affermie par les constatations qu'il nous fut donné de faire, au cours des années igro et rgri, en collaboration avec le Professeur Mercien, alors Chef des Travaux de Zoologie à la Faculté des Sciences de.l'Université de Nancy (2).

Force est, néanmoins, de reconsidérer le problème, tenu pour résolu, à raison des données nouvelles apportées par Schaperclaus et Nrbelin.

Disons, d'abord, que nul ne croit plus que les épidémies brutales, foudroyantes puissent être provoquées par les Myxosporidies comme l'ont cru Von Linstow, Zopf, Henneguy et Dubois. Ce dernier a le mérite d'avoir établi l'existence d'une relation curieuse, au point de vue pathologique, entre le Gardon et l'Ecrevisse (3), mais s'est mépris sur la nature du parasite commun, si insuffisamment étudié et décrit par lui qu'on ne sait s'il s'agissait d'un Sporozoaire ou d'un Champignon (4).

Quant à Henneguy (5), il a découvert une Myxosporidie, dénommée par luí Thelohania Contejeani, qui vit entre les faisceaux musculaires de l'Ecre-

(1) Dr Drounv de Bouvurs : - Les repeuplements en Ecrevisses, p. 24 ;- BergerLevrault, Paris et Nancy, Igj6.

Pour tous les détails relatifs aux caractéristiques de la peste, à son extension, à ses causes présumées, se reporter à cet ouvrage ou à la récente et fort intéressante publication d'Ambrí et Larr : - Les Ecrevisses de France ; - chez les auteurs, Paris, 1935.

(2) Mrrarer et de Drourn DE Bouvinte : - La Lépidorthose sur les Gardons du lac de Nantua. - Comptes rendus des séances de l'Académie des Sciences, 3o janvier I91 I ; - Gauthier-Villars, Paris.

Meraner et pr Droun de Bouvmas : - La maladie des Gardons du lac de Nantua et la thérie de M. le Professeur R. Dubois sur l'agent de la peste des Ecrevisses. Revue des Baux et Forets, 19 Juin 1911, p. 375. - Laveur, Paris.

DB Dnoum dB Bouvnrs : - La Peste des Ecrevisses en France. - Extrait du IX' Congres international de Zoologie tenu à Monaco du 25 au-30 mars rgr3. Oberthur, Rennes, rgi4.

(3) Dubors : - La peste des Ecrevisses, - Congrès international d'Acquiculture et de Peche tenu à Paris du if au 19 Septembre xgoo; - Mémoires et Comptes-rendus des Séances, p. 56. - Challamel, Paris, 1900.

(4) Dusors :- Comptes-rendus des Sćances de l'Académie des Sciences, 20 Fóvrier 1911. - Gauthier-Villars, Paris.

(5) Hennour : Myxosporidies parasites des muscles do quelques Crustacés Décapodes. - Annales de Micrographie, IV, 1891;1892; - Carré, Paris. 
visse. Elle désagrège les tissus aux dépens desquels elle se développe et en détermine la décoloration C'est ainsi que prennent naissance les taches d'un blanc caséeux ou crayeux assez fréquentes sous le test transparent du dessous de la queue. D'où le nom de Porzellankrankheit usité en Allemagne pour désigner une affection assez commune, semble-t-il, occasionnellement meurtrière, mais dont l'évolution est lente. Nous avons eu de fréquentes occasions, entre 1899 et 1914, d'observer des Crustacés éprouvés par elle ; dans la règle, ils ne succombent qu'à la longue. Nous avons noté qu'au dernier stade, assez souvent, que les pinces se détachent.

Actuellement, restent donc seuls en présence les champions du Bacterium pestis astaci Hofer et ceux de l'Aphanomyces astaci Schikora.

Nous fames des premiers; nous le restons pour les motifs qui vont être exposés, mais sans exclusivisme. Autrement dit nous ne croyons plus que les mortalités massives d'Ecrevisses d'avant ou d'après-guerre aient obligatoirement une même et unique cause.

Nous rejoignons ainsi le Professeur Kunstler qui a fait au Congrès International d'Aquiculture et de Pêche de 1900 cette judicieuse déclaration : - "Il paraît hors de doute que ce qui a été désigné sous la dénomination de peste de l'Ecrevisse est un ensemble de maladies et non une affection déterminée. Il y aura donc lieu de créer des diagnostics différentiels et de donner des noms différents à des maladies diverses. Quoi qu'il en soit, la maladie étudiée par M. Hofer... ayant reçu scientifiquement le nom de peste et son bacille étant désigné sous la dénomination de bacille de la peste, la qualification de peste de l'Ecrevisse sera réservée à cette maladie. " ( $\mathbf{r})$.

Il y a, en effet, des principes en matière de priorité. C'est en I 898 qu'une Bactérie particulière fut dénoncée comme germe de la peste (2) ; et, en I goo, les symptômes de l'infection par ce microparasite sont précisés à la suite d'observations poursuivies, en Juillet-Aout, durant sept semaines, dans le fleuve Woo, en Livonie (3). Ils consistent surtout dans une attitude singulière des Ecrevisses malades qui, se hissant sur leurs pattes raidies, marchent comme sur des échasses ; des convulsions suivent, dites clownesques, car elles évoquent les mouvements saccadés, désarticulés des acrobates ; enfin, après des crampes agoniques, la mort survient par paralysie du coeur.

ll se déduit de là que Scrikora, en 1903 (4) n'avait pas licence de dénom-

(1) Congrès international d'Acquiculture el de Peche tenu à Paris du I4 au r9 septembre 1900. - Mémoires et Comptes rendus des séances, p. 59. - Challamel, Paris, r 931 .

(2) Horer : - Uber die Krebspest : - Allgemeine Fischerei Zeitung, ${ }^{\text {er }}$ Septembre 1898. - Augsbourg.

(3) HoFen : - Recherches sur la peste des Ecrevisses en Russie. - Congrès international d'Aquiculture et de Peche tenu a Paris du 14 au ig Septembre 19oo. Mémoires et Comptes Rendus des séances, p. 39. - Challamel, Paris, rgor.

(4) Schirura : - Uber die Krebspest und ihren Erreger. - Fischerei Zeitung, année ı $903, n^{\circ} 23$, p. $353 ;-$ Neudam. 
mer peste la maladie par lui attribuée à un Champignon Oomycète de la famille des Saprolégniées.

Mais inutile d'insister sur ce point ; ce qui importe, en effet, est d'apporter quelque clarté dans un débat trop longtemps confus. Pour ne pas s'exposer à des généralisations aventureuses, le mieux serait de différencier, au moins provisoirement, une bacillose de l'Ecrevisse, dont l'agent est Bacillus astaciperda Lehmann-Neumann (r), et une mycose de l'Ecrevisse due à Aphanomyces astaci Schikora, l'une ou l'autre de ces deux maladies paraissant susceptible de dépeupler, en quelques jours, étangs, lacs ou biefs de cours d'eau. En tout cas, dans ce qui suit, nous observerons cette distinction.

Exposons maintenant les raisons pour lesquelles nous ne croyons pas qu'on puisse révoquer en doute, - car c'est à cela qu'on en vient, l'existence du $B$. astaciperda non plus que sa nocivité, au moins occasionnelle.

Cette Bactérie, MM. Mercier et moi l'avons, en effet, après Horeṇ, püehn (2), Weber (3) et Von Liphardet (4), isolée et cultivée à trois reprises.

La première fois, durant l'été de Igıo, elle fut trouvée dans le cœur et le foie de Gardons du lac de Nantua (Ain), victimes d'une épidémie. Certains d'entre eux présentaient un hérissement local des écailles caractérisant la lépidorthose, alors considérée comme imputable au $B$. astaciperda. La détermination fut vérifiée par Hofkr. Des doses frès faibles ( $1 /$ ro $^{\circ}$ de centìmètre cube) de bouillon de culture, injectées à des Ecrevisses pêchées dans un ruisseau de Lorraine où la peste n'avait jamais sévi, les firent périr en 5 à 7 heures avec les convulsions caractéristiques de l'astacobacillose. Par ailleurs, des inoculations intramusculaires ou intrapéritonéales ( $1 / 4$ à r/2 cc.) pratiquées sur des Gardons sains, amenèrent leur mort entre le troisième et le treizième jours consécutifs avec rebroussement plus ou moins accentué des écailles.

C'est dire que recevait entière confirmation la découverte de Plehn relative à l'identité d'agent pour deux maladies éprouvant, l'une les Poissons blancs, l'autre les Crustacés.

D'après Schapercíaus, la collaboratrice d'Hofer, qui soutenait encore cette identité en 1924 (5) n'en était plus convaincue en 1926. Sur quoi se

(1) Comme Schaperchaus, nous préférons ccttc dénomination en ce qu'elle n'est nullement tendancieuse.

(2) PhEHN : - D:e Schuppenstraubung der Weissfische verursacht durch das Krebspestbakteium ; - Allgemeine Fischerei Zeitung, ier Février 1902, p. 40. Augsbourg.

(3) Webre : - Zur Atiologie der Krebspest. - Arheilen aus äem kaiserlichen Gesundheitsamte, XV, p. 221 ; - Springer, Berlin, 1898 .

(4) Etudes expérimentales, morphologiques et biologiques, sur le. $B$. pestis astaci. - Comptes rendus sommaires du Congrès international de Peche et Pisciculture tenu à Saint-Pétersbourg en Février 19o2. - Revue Intcrnationale de Pêche et de Pisciculture, Décembre Igo2, p. 4. - Sant-Pétersbourg.

(5) Plehr : - Praktikum der Fischkrankheiten. - Stuttgart, 1934. 
fonde ce changement d'opinion, dont nous ignorons les motifs et qui n'a pas fait l'objet d'une publication émanant directement de l'intéressée ? Celle-ci ne se serait-elle pas bornée à reconnaître que la lépidorthose n'est pas une maladie suffisamment caractérisée, autrement dit que plusieurs infections bactériennes peuvent amener chez les Cyprinides le même phénomène du redressement des écailles ( $\mathrm{I}$ ).

En tout état de cause, après les constatations faites en collaboration avec Mercier, nous ne pouvons douter qu'en igro, une Bactérie, reconnue par Hofer identique au B. astaciperda, infestait les Gardons du lac de Nantua (2) et, inoculée à l'Ecrevisse, déterminait une mort rapide précédée de convulsions tétaniques.

L'année suivante, en Février-Mars toutes les Ecrevisses en cours d'élevage à l'établissement de pisciculture de Bellefontaine (Meurthe-et-Moselle) (3), dépendance de l'Ecole Nationale des Eaux et Forêts dont nous avions la gestion, moururent en quelques semaines. Les recherches bactériologiques amenèrent à incriminer le $B$. astaciperda, mais les symptômes considérés comme propres à la peste ne furent pas suffisamment nets pour que le diagnostic fût assuré. D'ailleurs les sujets malades, parqués dans des bassins à parois cimentées, y vivaient dans des conditions passablement différentes de celles de la nature. Il n'en reste pas moins que, pour la seconde fois, la Bactérie découverte par Hofer était identifiée en France.

La troisième occasion de vérifier la thèse du savant munichois ne se fit pas attendre.

Au début de l'été, mission nous fut donnée, par la Direction Générale des Eaux et Forêts, de rechercher les causes du dépeuplement du Gour de Champlives dans le Département du Doubs. Ce ruisseau présente cette particularité de couler dans un bassin fermé, car à sept kilomètres environ de sa source, il s'infiltre dans le sol. Isolé de la sorte, il était resté riche en Ecrevisses à pattes blanches jusqu'au printemps de rgir. A ce moment, les Crustacés commencèrent à périr nombreux au voisinage de la perte ; puis

(I) D'après un ouvrage allemand tout récemment paru, le hérissement des écailles est toujours le symptôme d'une affection grave : altération des reins, anémie, infection bactérienne. - lilustriertes Fischerei Lexikin, p. 148. Neumann, Neudamm, 1936 .

(2) Précisions aujourd'hui, pour être complet, que les Ecrevisses dont tous les touristes ne manquent pas de se régaler à leur passage à Nantua, étaient, en 19io, importées d'Allemagne ; nous avons eu communication, à l'époque, du relevé des arrivages. Les Crustacés indigènes ont été, en effel, anénntis entre y $88_{0}$ et s8go. Dans. ces conditions, la présence du $B$. astaciperda dans l'organisme des Gardons du lac n'avait rien d'énigmatique.

(3) De Drour de Bouvirt: : - Nole sur un eśsai d'elevage de l'Ecrevisse à pattes rouges. - Comptes rendus des Séances de la Société de Biologie, LXVII, p. 745 . Maretheux, Paris, rgog.

De Dhor in de Borvilue : - Sur un essai d élevage de l'Fcrevisse à pattes rouges. Comptes rendus des Séances de la Société de Biologie, LXIX, p. 646. - Maretheux, Paris, rgio.

De Drouix de Bouville : - La pratique des repeuplements en Ecrevisses, pp. i6-i 7 . - Berger-Levrault, Paris et Nancy, rgio. 
la maladie gagna assez lentement vers l'amont, marquant, en particulier, un arrét de quelque durée au pied d'un barrage de moulin.

A notre arrivée sur les lieux, dans les premiers jours de Juin, nous eumes la surprise de faire des constatations exactement similaires de celles effectuées par HoFen en ${ }^{-}$Livonie, treize ans auparavant. Pas un trait ne manquait au tableau. En particulier les sujets agonisants se déplaçaient péniblement, le corps surélevé sur les pattes roides, ce après quoi, perdant l'équilibre, ils restaient sur le dos ou sur le flanc, les membres agités quelque temps de convulsions tétaniques.

Nous ne croyons pas qu'il y ait eu souvent manifestations de bacillose pure s'offrant de pareille manière à l'observation. Nous disons : bacillose pure, car tous les Crustacés pestiférés rapportés au laboratoire s'y révélèrent, après examen minutieux, indemnes de parasites tels que Champignons, Infusoires, Sporozoaires.

Leur état de santé apparente était même tel que les premiers essais d'ensemencement de cultures, en partant du sang, ne donnèrent absolument rien. Mais, Mercien ayant eu l'idée de placer sur la gêlatine le coeur tout entier d'un individu sacrifié, le $B$. astaciperda se laissa enfin déceler. Toutes vérifications utiles fảites, la certitude fut acquise que la Bactérie quí, l'année précédente, avait été découverte dans les Gardons de Nantua, se retrouvait chez les Ecrevisses de Champlives, lesquelles, succombaient à son attaque avec tous les symptómes caractéristiques de la maladie alors désignée sous le nom de peste.

Après avoir vu ce que nos yeux ont vu, il nous est rigoureusement impossible d'admettre que toutes les épidémies sur les Ecrevisses de 1876 à ı 196 , puissent se laisser ramener à une seule et même affection imputable à un Oomycète. Mais, nous n'irons pas dire pour autant que toutes sont d'origine bactérienne.

Il faut, aujourd'hui surtout où la Science se montre d'autant plus réservée dans ses conclusions que ses investigations se font davantage précises, tenir compte de toutes les recherches bien faites, et ne généraliser qu'à bon escient. Que Scmorn et Horne se soient, il y a un quart de siècle, passionnés, qui pour un Champignon, qui pour un Bacille, cela est humain, Mais leurs témoignages contradictoires, dont il n'y a pas lieu de suspecter la loyauté, valent pour les faits.observés de part et d'autre, sans plus. Ce qui, en conformité des suggestions émises dès rgoo par Kunstrkn, conduit à admettre, jusqu'à plus ample informé, l'existence de deux infections distinctes meurtrières pour l'Ecrevisse à savoir : une astacobacillose et une astacomycose.

Reste la question du diagnostic différentiel, question épineuse. Car si les deux parasites différents à tenir pour responsables de maladies également redoutables déterminent chez leurs victimes des lésions similaires, les réactions seront analogues.

Cependant la bacillose ne se laisse-t-elle pas reconnattre à cette curieuse 
ataxie locomotrice des Crustacés près de leur fin, qui se soulevant sur leurs pattes thoraciques comme ankylosées aux jointures, titubent péniblement ? Il y a, aussi, les convulsions clownesques qui suivent, enfin la rigidité cadavérique. Ces symptômes ont été donnés comme distinctifs, par HoFer, dès le début de ses travaux ; il les a relevés en particulier, en rgoo. sur les Ecrevisses du Woo; personnellement, nous les avons notés très nets, sur celles du Gour de Champlives en rgI I. Au surplus, quand on inocule des cultures de $B$. astaciperda à des sujets sains, on reproduit les symptômes en question comthe il a été relaté plus haut.

Reportons-nous maintenant au dernier travail de Schaperclaus (I) il semble que l' $A$. astaci affaiblisse les articulations au lieu de les raidir. Citons : - "Les Crustacés, incapables de se tenir sur leurs pattes, tombent sur le dos. Si, à ce moment, on prend un sujet malade et qu'on le sorte de l'eau, en position normale, les pinces pendent au-dessus du corps comme celles d'un individu mort. Dans la nature, les Ecrevisses atteintes s'efforcent de gagner le rivage ; souvent leurs pattes se détachent au cours de cette tentative. "Nous n'avons rien remarqué de semblable dans les cas de bacillose que nous avons pu étudier.

Toutefois, les différences éventuelles de comportement n'ont pas attiré suffisamment l'attention au moment où les occasions d'observer les mortalités d'Ecrevisses étaient fréquentes. Il serait imprudent d'affirmer quoi que ce soit à leur égard mais il est permis de signaler que certaines discordances se laissent soupconner entre l'action sur l'hôte du Bacille d'Hofer et celle du Champignon de Schixora. Le premier aurait une influence paralysante et tétanisante sinon absolument propre, du moins particulièrement accentuée.

En terminant, il n'est pas inutile d'indiquer les motifs pour lesquels le $B$. astaciperda a été découvert si tardivement et est si difficile à isoler. Redi-

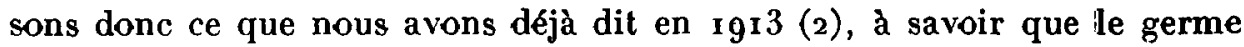
en question est loin d'abonder dans l'organisme des Crustacés qu'il infeste ; sa nocivité tenant moins à sa pullulation qu'à la virulence des toxines sécrétées. En outre, il faut le chercher là où il se localise de préférence, à savoir dans la musculature. Car Hofer nous écrivait en rgI I : - "Je puis vous informer qu'il n'est pas du tout rare de ne trouver aucune Bactérie dans le sang du cœur. ... J'effectue surtout les prélèvements à fins de culture dans les pinces de l'Ecrevisse ; après arrachage, la section ayant été passée à la flamme, j'introduis par là le fil de platine et l'enfonce profondément. C'est en opérant ainsi qu'on a les meilleures chances de réussite ".

Peut-être, en recourant à cette technique, parviendrait-on à remettre dans la circulation l'agent devenu introuvable, de l'astacobacillose.

(r) La cause des pestes d'Ecrevisses:- Bullelin, $\mathrm{n}^{\circ}$ 10, Novembre rg36, p. 117 .

(2) La peste des Ecrevisses en France. - l. cit. p. 690. 